\section{Impact of FLT3-ITD length on prognosis of acute myeloid leukemia}

The FMS-like tyrosine kinase-3 internal tandem duplication (FLT3-ITD) is one of the most frequent mutations found in acute myeloid leukemia (AML) patients, with a frequency of $20-30 \%$. The sizes of ITDs vary dramatically. ${ }^{1}$ The FLT3-ITD consists of in-frame insertions of duplicated sequences, most of them $(70 \%)$ are located in the juxtamembrane domain (JMD), while the remaining are in tyrosine kinase domain 1 (TKD1). ${ }^{2,3}$ Currently, it is well known that TKD1-ITDs, while significantly longer than JMD-ITDs, are associated with worse outcome. ${ }^{3-5}$ However, previous studies failed to exclude the TKD1$\mathrm{ITD}^{+}$patients for the correlation analysis of ITD length and prognosis, resulting in potential statistical interference. $^{6-10}$ On the other hand, structural studies have demonstrated that only 15 base pair (bp) ITD insertions in JMD can disrupt the FLT3 kinase auto-inhibition conformation, thus promoting ligand-independent FLT3 receptor dimerization, and leading to constitutive autophosphorylation and activation of downstream survival and proliferation signaling. ${ }^{11,12}$ To date, there is no clear consensus on whether the length of ITD affects the clinical outcome of patients with JMD-ITD. ${ }^{6-10}$ Therefore, in this study, we specifically selected JMD-ITDs to examine the impact of the variability in ITD length on biological phenotype, and correlated this variability with clinical outcome in a cohort of adult AML patients with single ITD mutations.

We selected 89 adult de novo AML patients (excluding acute promyelocytic leukemia) with FLT3-ITD mutations retained in our tissue bank between September 2010 and October 2015 (Online Supplementary Figure S1). Every selected patient had single ITD insertion. All 89 ITDs were sequenced and quantified using an ABI 3730 automated DNA sequencer ${ }^{13}$ (Figure 1A). The lengths of the 89 ITDs ranged from 6 to $90 \mathrm{bp}$, with a median of $39 \mathrm{bp}$ (13 amino acids) (Online Supplementary Table S1). In addition, the genomic localization of the ITD varied: most ITDs $(77.5 \%)$ were located in the JM-Z region $(n=69)$, $19.1 \%(n=17)$ of ITDs were located in the hinge region, only 3 ITDs (3.4\%) were located in JM-S region.

We further explored whether ITD length impacted clinical outcome. Using the median ITD length of $39 \mathrm{bp}$ as cut off, all 89 patients were divided into two groups. Interestingly, there was no relationship between ITD length and the FLT3-ITD allelic ratio or age (Figure 1B and $\mathrm{C}$ ). In our results, the group of larger ( $\geq 39 \mathrm{bp}$ ) ITDs had higher median white blood cell (WBC) counts at diagnosis $(P=0.044)$ and a trend of more female patients (30 of 48 vs. 18 of 51; $P=0.079$ ) (Online Supplementary Table S2). Between these two groups, there were no statistical differences according to marrow blast counts, hemoglobin, platelet, age, treatment of allogeneic hematopoietic stem cell transplantation (allo-HSCT), cytogenetic distribution or mutation status of NPM1, $C E B P A$, and DNMT3A genes. We found that treatment outcome was poorer for patients with larger ITDs $(\geq 39$ bp) compared to patients with smaller ITDs $(<39 \mathrm{bp})$ [overall survival (OS) rate at 3 years: $27.1 \%$ vs. $61.2 \%$; $P=0.004$ ] (Figure 1D). Likewise, shorter progression-free survival (PFS) was associated with increased ITD length $(P=0.006)$. The 3 -year PFS rates were $47.0 \%$ and $21.2 \%$ for patients with small and large ITDs, respectively (Figure 1E). Importantly, adjusted by other confounders (Table 1), multivariate Cox regression model analysis indicated that larger ITD size ( $\geq 39 \mathrm{bp}$ ) was an independent prognostic factor for inferior outcomes with Hazard Ratios (HR) of 2.27 ( $P=0.014)$ for OS, and $2.06(P=0.014)$ for PFS. Based on the power simulation, the sample size was sufficient to draw statistical conclusions (Online Supplementary Table S3 and Online Supplementary Figure $S 2)$. In addition, ITD allelic ratio is considered to be an independent factor for worse outcome and is cited in the 2017 edition of European LeukemiaNet (ELN). ${ }^{14}$ Similar results were confirmed in our study (Online Supplementary Table S4 and Online Supplementary Figure S3), while age was excluded out of the multivariate analysis due to the equilibrium of age distribution for these two groups (Table 1 and Online Supplementary Tables S2 and S5).

Next, we examined the biological function of different ITD lengths. From the $89 \mathrm{ITD}^{+}$patients, we chose the median size $39 \mathrm{bp}$ as the cut-off value to screen the "long ITD" and "short ITD" sequences, and we chose two pairs of ITD sequences from the JM-Z and hinge regions. Each pair of ITDs differed in length, but was located in the same or adjacent insert site (Figure 2A). Each of the four ITDs were then amplified and individually sub-cloned into a lentiviral vector co-expressing green fluorescent protein (GFP). Murine myeloid 32D cells stably expressing wild-type FLT3 or each of the four FLT3-ITD constructs were generated by lentiviral transduction, and comparable surface expression of FLT3 in each clone was confirmed by FACS analysis (data not shown). As expect-

Table 1. Multivariate Cox regression models of association between length of internal tandem duplication (ITD) and survival.

\begin{tabular}{|c|c|c|c|c|}
\hline & \multicolumn{2}{|c|}{ Overall survival } & \multicolumn{2}{|c|}{ Progression-free survival } \\
\hline & Hazard ratio (95\% Cl) & P & Hazard ratio (95\% Cl) & $P$ \\
\hline ITD length ( $\geq 39$ vs. $<39 \mathrm{bp}$ ) & $2.14(1.12 \sim 4.08)$ & 0.022 & $2.06(1.18 \sim 3.61)$ & 0.012 \\
\hline ITD ratio $(\geq 0.32 v s .<0.32)$ & $1.94(1.03 \sim 3.67)$ & 0.041 & $1.43(0.83 \sim 2.49)$ & 0.199 \\
\hline Sex (male $v s$. female) & $0.61(0.33 \sim 1.15)$ & 0.127 & $0.70(0.40 \sim 1.24)$ & 0.221 \\
\hline \multicolumn{5}{|l|}{ Cytogenetic risk group } \\
\hline Favorable risk & Reference group & & Reference group & \\
\hline Intermediate risk & $7.14(0.96 \sim 53.03)$ & 0.055 & $3.78(1.15 \sim 12.42)$ & 0.029 \\
\hline High risk & $12.18(1.19 \sim 124.89)$ & 0.035 & $4.54(0.95 \sim 21.74)$ & 0.058 \\
\hline DNMT3A (mut $v s$. unmut) & $2.29(1.16 \sim 4.50)$ & 0.017 & $2.21(1.22 \sim 4.02)$ & 0.009 \\
\hline HSCT under CR1 (yes vs. no) & $0.30(0.15 \sim 0.61)$ & $<0.001$ & $0.24(0.13 \sim 0.45)$ & $<0.001$ \\
\hline
\end{tabular}

HSCT: hematopoietic stem cell transplantaion; CR1: first complete remission; CI: Confidence Interval; mut: mutated; unmut: unmutated. 
ed, the four ITD infected 32D lines but not the wild-type FLT3 control, proliferated and survived under IL-3 deprivation; this is consistent with previous studies showing ITD mutations conferring growth factor-independent cell growth. ${ }^{11,12}$ Interestingly, cells expressing the long FLT3ITD sequences (Mut 2 and Mut 4) had higher growth factor-independent proliferative capacity regardless of the insertion localization compared with the short FLT3-ITD clones (Figure 2B).

Next, we analyzed the constitutive tyrosine phosphorylation of FLT3-ITDs, and their downstream genes like STAT5, Akt, using specific phosphorylation antibodies. As we expected, the longer FLT3-ITDs showed stronger phosphorylation signals, regardless of whether the insertions were located in JM-Z or hinge regions (Figure $2 \mathrm{C}$ ). We assessed the effect of ITD length on sensitivity to the second-generation FLT3-kinase inhibitor quizartinib (AC220). AC220 is a promising compound which is currently under evaluation in a multicenter phase III clinical trial. $^{15}$ 32D cells transformed with the Mut1, Mut2, Mut3, and Mut4 FLT3-ITDs were exposed to various doses of AC220 for three days. Although nanomolar concentration of AC220 could effectively inhibit growth of $32 \mathrm{D}$ cells harboring any of the ITD variations, cells harboring the longer ITDs were less sensitive than those with shorter ITDs to AC220 treatment, as shown by

A

FLT3-ITD Mutation in Juxtamembrane Domain

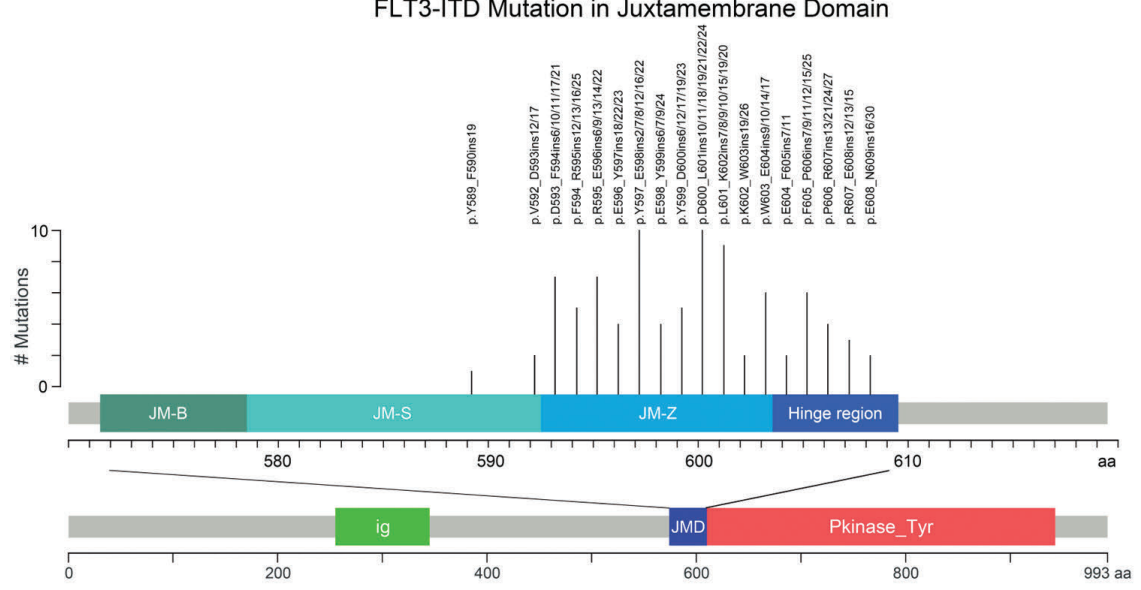

B

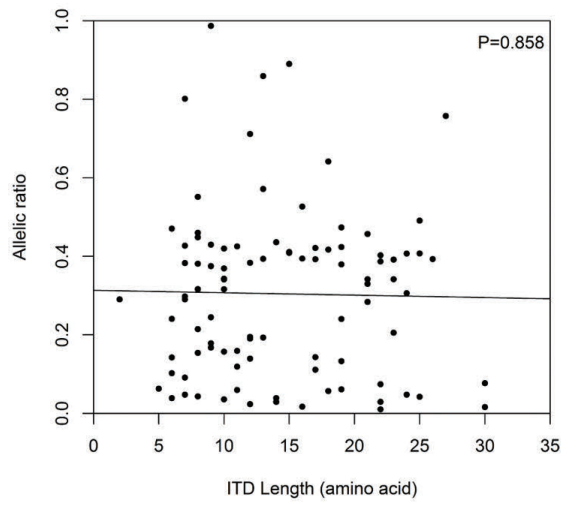

D

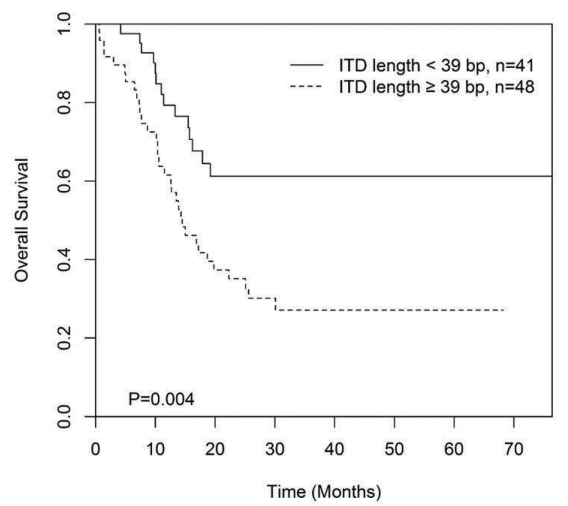

C

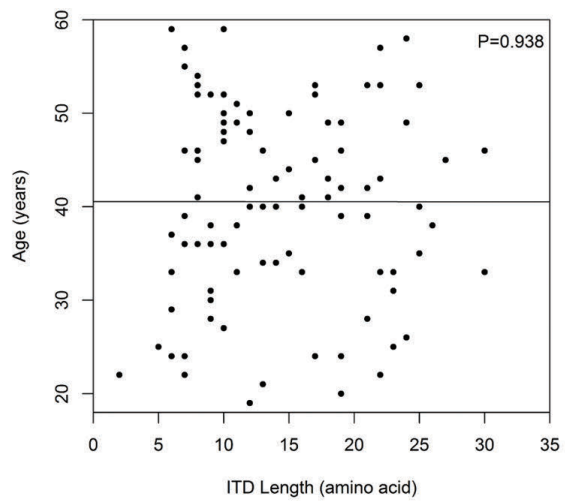

E

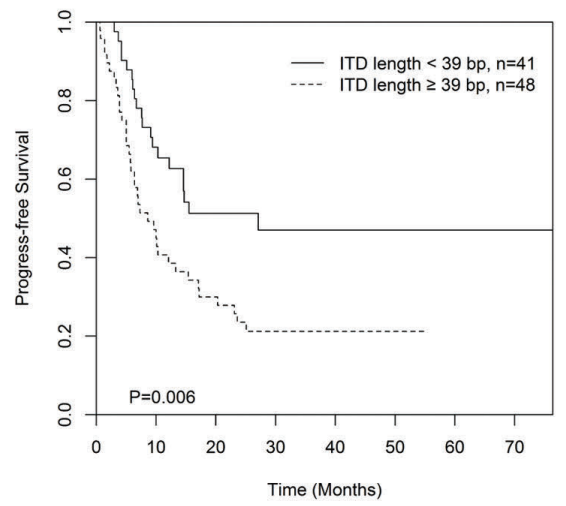

Figure 1. Survival analysis for FLT3-ITD-mutated acute myeloid leukemia (AML) according to length of internal tandem duplication (ITD). (A) FLT3 and the locations of ITD mutations in the juxtamembrane domain (JMD). (B) ITD length and allelic ratio. No correlation was observed between ITD length and allelic ratio by Spearman correlation coefficient $(r=0.018$, $P=0.858$ ). (C) ITD length and age. No correlation was observed between ITD length and age by Spearman correlation coefficient $(r=0.008$, $P=0.938$ ). (D) Estimated 3-year overall survival (OS) for ITD length equal to or longer than 39 bp versus shorter than 39 bp. (E) Estimated 3-year progression-free survival (PFS) for ITD length equal to or longer than 39 bp versus shorter than 39 bp. Group differences for both $\mathrm{OS}$ and PFS were analyzed by log-rank tests. 
both proliferation $\left(\mathrm{Mut}_{\mathrm{IC} 50}=0.446 \mathrm{nM}\right.$ vs. Mut $2_{\mathrm{IC50}}=$ $0.836 \mathrm{nM}$, Mut $_{\mathrm{IC} 50}=0.530 \mathrm{nM}$ vs. Mut4 $\left.{ }_{\mathrm{IC} 50}=0.847 \mathrm{nM}\right)$ (Figure 2D) and apoptosis (Figure 2E); the same conclusion was also made for sorafenib or midostaurin treatment (Online Supplementary Figure S4). These results indicated that longer ITDs are associated with higher FLT3 kinase activity. This is in line with the proliferative advantage (Figure 2B) and higher FLT3 autophosphorylation (Figure 2C).

Taken together, although there is considerable disagreement regarding the prognostic impact of FLT3-ITD length; our results established a biological link between the ITD length in JMD and FLT3 kinase activity. Mechanistically, it is possible that the longer insertion in the JMD may cause more significant disruption of its autoinhibitory function. ${ }^{11,12}$ In addition, our clinical data showed that patients with longer ITDs had significantly worse OS and PFS rates, and ITD longer than $39 \mathrm{bp}$ was an independent prognostic factor in multivariate Cox regression models. These findings may help to stratify patients when considering therapeutic combinations based on tyrosine kinase inhibitors.

Song-Bai Liu, ${ }^{*}$ Hao-Jie Dong, ${ }^{2 *}$ Xie-Bing Bao, $3,4,5^{*}$

Qiao-Cheng Qiu, ${ }^{3,4,5^{*}}$ Hong-Zhi Li, ${ }^{6}$ Hong-Jie Shen, ${ }^{3,4,5}$

A

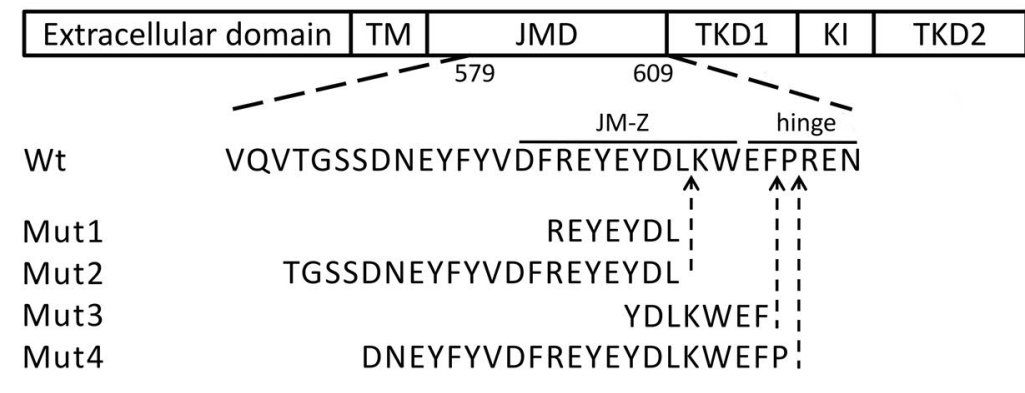

B

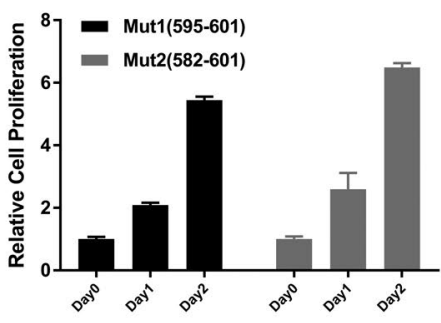

C

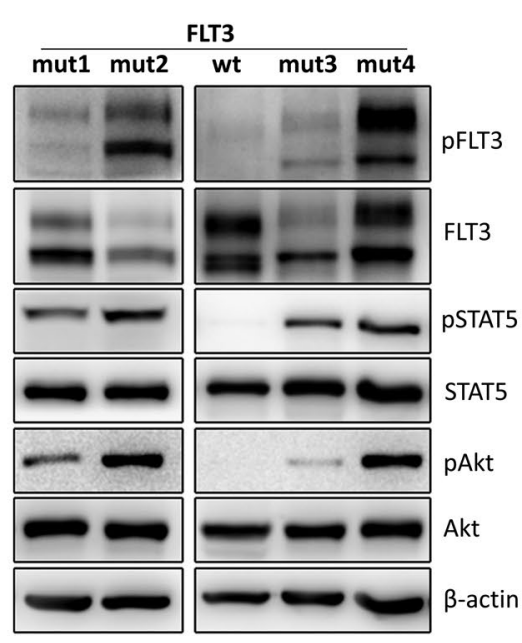

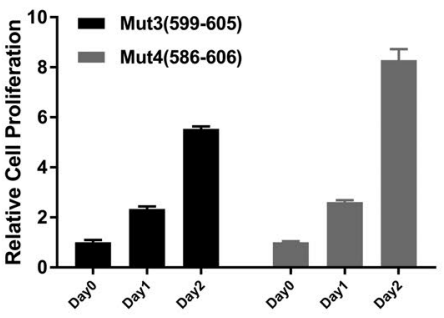

D
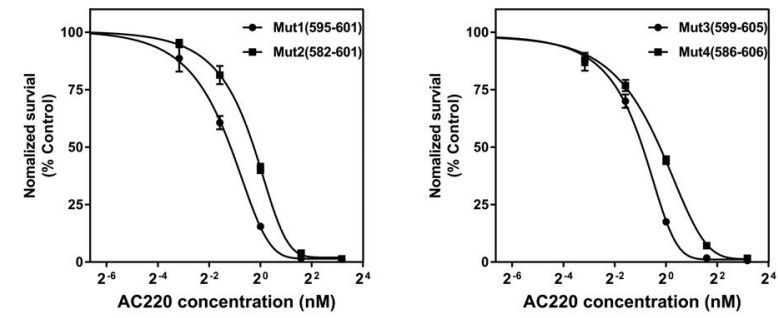

$\mathrm{E}$
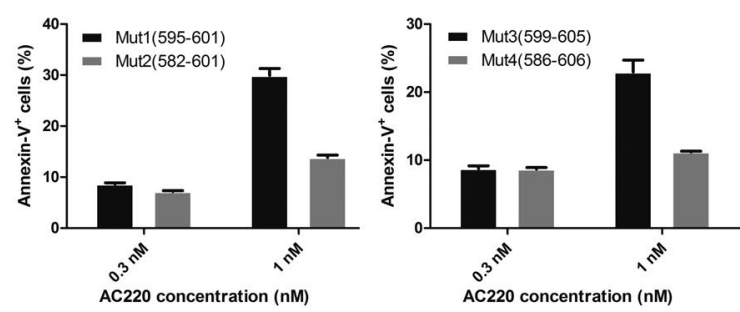

Figure 2. Comparison of biological properties of FLT3-ITDs with long and short internal tandem duplication (ITD) insertion length. (A) Localization and inserted amino acids of the 4 ITD mutations in JMZ (Mut1, Mut2) and hinge region (Mut3, Mut4). (B) Cell proliferation capacity of FLT3-ITD mutations in JMZ (left) and hinge region (right) without IL-3 assayed by Cell Titer-Glo. The relative cell proliferation was normalized to the value of each group at day 0 . (C) Phosphorylation of FLT3 (Y589/591), STAT5, and Akt of 4 FLT3-ITD cell strains with ITD insertions in JMZ (left) and hinge region (right), was determined by immunoblotting. (D) Dose response curves of mutant FLT3-ITD clone cell proliferation in the presence of AC220. (E) Induction of apoptosis in FLT3-ITD transduced 32D cell strains with AC220. Survival rate was determined by flow cytometry of DAPI and Annexin-V stained cells. 


\author{
Zi-Xuan Ding, 3,4,5 Chao Wang, 3,4,5 Xiao-Ling Chu, 3,4,5 \\ Jing-Qiu Yu, 3,4,5 Tao Tao, 3,4,5 Zheng Li, ${ }^{3,4,5}$ Xiao-Wen Tang, 3,4,5 \\ Su-Ning Chen, ${ }^{3,4,5}$ De-Pei Wu, ${ }^{3,4,5^{*}}$ Ling L $i^{2 \#}$ and Sheng-Li \\ $X u e^{3,4,5^{*}}$
}

*These authors contributed equally to this work.

'Suzhou Key laboratory for medical biotechnology, Suzhou Vocational Health College, Suzhou, China; ${ }^{2}$ Department of Hematological Malignancies Translational Science, Beckman Research Institute, City of Hope National Medical Center, Duarte, CA, USA; Jiangsu Institute of Hematology, The First Affiliated Hospital of Soochow University, Suzhou, China; ${ }^{4}$ Institute of Blood and Marrow Transplantation, Suzhou, China; ${ }^{5}$ Collaborative Innovation Center of Hematology, Soochow University, Suzhou, China and ${ }^{6}$ Department of Molecular Medicine, Beckman Research Institute, City of Hope National Medical Center, Duarte, CA, USA

Funding: this work was supported by grants from the National Natural Science Foundation of China (Grant No. 81470296, 31701198), Jiangsu Provincial Medical Youth Talent Program (Grant No. QNRC2016719, QNRC2016771), Natural Science Foundation of Jiangsu Province of China (Grant No. BK20170386), a C class sponsored project of Jiangsu provincial Six Talent Peaks (Grant No. 2016-WSN-123), and the China National Clinical Key Subject Project. This work was supported in part by the National Institutes of Health under award number R00CA184411, R01HL141336, V Scholar Research Award, the Stop Cancer Research Career Development Award and the Gehr Family Center for Leukemia Research (to L.L.). Research reported here includes work performed in the Analytical Cytometry Core at City of Hope supported by the National Cancer Institute of the National Institutes of Health under award number P30CA33572. The content is solely the responsibility of the authors and does not necessarily represent the official views of the National Institutes of Health.

Acknowledgments: we also thank Lynn Smith for help in manuscript editing and proofreading.

Correspondence: WSBSL5806@163.com or lingli@coh.org or slxue@suda.edu.cn

doi:10.3324/haematol.2018.191809

Information on authorship, contributions, and financial \& other disclosures was provided by the authors and is available with the online version of this article at www. haematologica.org.

\section{References}

1. Nakao M, Yokota S, Iwai T, et al. Internal tandem duplication of the flt3 gene found in acute myeloid leukemia. Leukemia. 1996;10(12):1911-1918.

2. Breitenbuecher F, Schnittger S, Grundler R, et al. Identification of a novel type of ITD mutations located in nonjuxtamembrane domains of the FLT3 tyrosine kinase receptor. Blood. 2009;113(17):4074-4077.

3. Kayser S, Schlenk RF, Londono MC, et al. Insertion of FLT3 internal tandem duplication in the tyrosine kinase domain-1 is associated with resistance to chemotherapy and inferior outcome. Blood. 2009; 114(12):2386-2392.

4. Schlenk RF, Kayser S, Bullinger L, et al. Differential impact of allelic ratio and insertion site in FLT3-ITD-positive AML with respect to allogeneic transplantation. Blood. 2014;124(23):3441-3449.

5. Arreba-Tutusaus P, Mack TS, Bullinger L, et al. Impact of FLT3-ITD location on sensitivity to TKI-therapy in vitro and in vivo. Leukemia. 2016;30(5):1220-1225

6. Gale RE, Green C, Allen C, et al. The impact of FLT3 internal tandem duplication mutant level, number, size, and interaction with NPM1 mutations in a large cohort of young adult patients with acute myeloid leukemia. Blood. 2008;111(5):2776-2784

7. Stirewalt DL, Kopecky KJ, Meshinchi S, et al. Size of FLT3 internal tandem duplication has prognostic significance in patients with acute myeloid leukemia. Blood. 2006;107(9):3724-3726.

8. Meshinchi S, Stirewalt DL, Alonzo TA, et al. Structural and numerical variation of FLT3/ITD in pediatric AML. Blood. 2008; 111(10):4930-4933.

9. Kusec R, Jaksic O, Ostojic S, Kardum-Skelin I, Vrhovac R, Jaksic B. More on prognostic significance of FLT3/ITD size in acute myeloid leukemia (AML). Blood. 2006;108(1):405-406.

10. Kim Y, Lee GD, Park J, et al. Quantitative fragment analysis of FLT3ITD efficiently identifying poor prognostic group with high mutant allele burden or long ITD length. Blood Cancer J. 2015;5:e336.

11. Griffith J, Black J, Faerman C, et al. The structural basis for autoinhibition of FLT3 by the juxtamembrane domain. Mol Cell. 2004; 13(2):169-178

12. Chan PM. Differential signaling of Flt3 activating mutations in acute myeloid leukemia: a working model. Protein Cell. 2011;2(2):108-115.

13. Meshinchi S, Woods WG, Stirewalt DL, et al. Prevalence and prog nostic significance of Flt3 internal tandem duplication in pediatric acute myeloid leukemia. Blood. 2001;97(1):89-94.

14. Dohner H, Estey E, Grimwade D, et al. Diagnosis and management of AML in adults: 2017 ELN recommendations from an international expert panel. Blood. 2017;129(4):424-447.

15. Larrosa-Garcia M, Baer MR. FLT3 Inhibitors in Acute Myeloid Leukemia: Current Status and Future Directions. Mol Cancer Ther. 2017;16(6):991-1001. 